Homology, Homotopy and Applications, vol.3(2), 2001, pp.407-415

\title{
THE TANGENT BUNDLE OF AN ALMOST-COMPLEX FREE LOOPSPACE
}

\author{
JACK MORAVA \\ (communicated by Gunnar Carlsson)
}

\begin{abstract}
The space $L V$ of free loops on a manifold $V$ inherits an action of the circle group $\mathbb{T}$. When $V$ has an almost-complex structure, the tangent bundle of the free loopspace, pulled back to a certain infinite cyclic cover $\widetilde{L V}$, has an equivariant decomposition as a completion of $\mathbf{T} V \otimes(\oplus \mathbb{C}(k))$, where $\mathbf{T} V$ is an equivariant bundle on the cover, nonequivariantly isomorphic to the pullback of $T V$ along evaluation at the basepoint (and $\oplus \mathbb{C}(k)$ denotes an algebra of Laurent polynomials). On a flat manifold, this analogue of Fourier analysis is classical.
\end{abstract}

The purpose of this note is to show that the study of the equivariant tangent bundle of a free smooth loopspace can be reduced to the study of a certain finitedimensional vector bundle over that loopspace - at least, provided the underlying manifold has an almost-complex structure (e.g. it might be symplectic), and if we are willing to work over a certain interesting infinite-cyclic cover of the loopspace. The first section below summarizes the basic facts we'll need from equivariant differential topology and geometry, and the second is a quick account of the universal cover of a symmetric product of circles, which is used in the third section to construct the promised decomposition of the equivariant tangent bundle. It is interesting that the covering transformations and the circle act compatibly on the tangent bundle of the covering, while their action on the splitting commutes only up to a projective factor.

\section{The free loopspace and its universal cover}

1.1 If $V$ is a connected compact almost-complex manifold of real dimension $2 n$, the space of smooth maps from the circle

$$
S^{1}=\{x \in \mathbb{C}|| z \mid=1\}
$$

to $V$ is an (infinite-dimensional) manifold $L V$, with local charts defined by the vector bundle neighborhoods of $[5 \S 13]$; the tangent space at the loop $\sigma$ is a vector

The author was supported in part by the NSF

Received May 14, 2001, revised August 7, 2001; published on September 13, 2001.

2000 Mathematics Subject Classification: 58Dxx; 53C29, 55P91

Key words and phrases: free loopspace, circle action, holonomy, polarization.

(C) 2001, Jack Morava. Permission to copy for private use granted. 
space

$$
T_{\sigma} L V=\Gamma_{S^{1}}\left(\sigma^{*} T V\right)
$$

of sections of the pullback of the tangent bundle along $\sigma$. The circle group $\mathbb{T}$ acts on $L V$ by rotating loops, and rotation through the angle $\alpha$ lifts to the complex-linear transformation

$$
\alpha_{*}: T_{\sigma} L V \rightarrow T_{\sigma \circ \alpha} L V
$$

which sends the section $v$ of $\sigma^{*} T V$ to the section

$$
\alpha_{*}(v)(\theta)=v(\theta+\alpha)
$$

of $(\sigma \circ \alpha) * T V$. The tangent bundle of $L V$ is thus an (infinite-dimensional) complex $\mathbb{T}$-equivariant vector bundle; in fact it is the free loopspace of $T V$. If $V$ is simplyconnected then the free loopspace will be connected; from now on I will assume this.

Connections on vector bundles pull back, so a connection $\nabla$ on the tangent bundle of $V$ pulls back to a connection on $\sigma^{*} T V$. Applied to the standard vector field $d / d \theta$ on the circle, the connection defines a derivation

$$
D_{\sigma}: T_{\sigma} L V \rightarrow T_{\sigma} L V
$$

on the tangent space. It is easy to see that the diagram

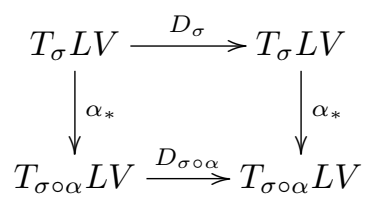

commutes, or in other words that

$$
\alpha_{*} D_{\sigma}=D_{\sigma \circ \alpha} \alpha_{*} .
$$

If $\Gamma_{j k}^{i}(x)$ are the connection coefficients in local coordinates at $x \in V$, then

$$
D_{\sigma} v^{i}(\theta)=\dot{v}^{i}(\theta)+\Gamma_{j k}^{i}(\sigma(\theta)) \dot{\sigma}^{k}(\theta) v^{j}(\theta)=\dot{v}^{i}(\theta)+B_{j}^{i}(\theta) v^{j}(\theta)
$$

and hence

$$
D_{\sigma \circ \alpha} \alpha_{*}(v)^{i}(\theta)=\dot{v}^{i}(\theta+\alpha)+B_{j}^{i}(\theta+\alpha) v^{j}(\theta+\alpha),
$$

which is just $\alpha_{*}\left(D_{\sigma} v\right)^{i}(\theta)$.

1.2 A Hermitian metric $h$ on $T V$ defines an inner product

$$
(v, w)=\int_{S^{1}}(v, w)_{h}(\theta) d \theta
$$

on $T_{\sigma} L V$. Given such a metric, let $\nabla$ be its associated connection; then [7 III $\left.\S 2.1\right]$

$$
\left.\left(D_{\sigma} v, w\right)+\left(v, D_{\sigma} w\right)=\int_{S^{1}} d(v, w)_{h}(\theta)\right)=0
$$


which is to say that $D_{\sigma}$ is a skew-adjoint differential operator acting on sections of a Hermitian vector bundle. The circle is compact and one-dimensional, so $D_{\sigma}$ is elliptic, with discrete (purely imaginary) spectrum.

If $C$ is a closed subset of this spectrum, let $\mathcal{P}_{C}$ be the projection onto the span of the eigenvectors with eigenvalues in $C$. When $C=\{h\}$ is a single eigenvalue, $\mathcal{P}_{h}$ will denote the projection onto the space of associated eigenvectors; thus

$$
\mathcal{P}_{C}=\sum_{c \in C} \mathcal{P}_{c}: T_{\sigma} L V \rightarrow T_{\sigma} L V
$$

if $C$ is finite.

If $v$ is an eigenvector of $-i D_{\sigma}$ with eigenvalue $h$, then for any integer $k$,

$$
D_{\sigma}\left(v(\theta) e^{i k \theta}\right)=D_{\sigma}(v(\theta)) e^{i k \theta}+i k v(\theta) e^{i k \theta}=i(h+k) v(\theta) e^{i k \theta},
$$

so $v(\theta) e^{i k \theta}$ will also be an eigenvector. The eigenvalues of $-i D_{\sigma}$ therefore fall into a (finite) set of equivalence classes in $\mathbb{R} / \mathbb{Z}$, and any $v \in T_{\sigma} L V$ has a Fourier-like decomposition

$$
v(\theta) \sim \sum_{k \in \mathbb{Z}} v_{k}(\theta) e^{i k \theta}
$$

with coefficients in the (finite-dimensional) vector space $\left.T_{\sigma} L X\right|_{[0,1)}$ spanned by eigenvectors of $-i D_{\sigma}$ with eigenvalues in the half-open interval $[0,1)$. Since

$$
\alpha_{*} \circ D_{\sigma} \circ \alpha_{*}^{-1}=D_{\sigma \circ \alpha},
$$

eigenvectors of $D_{\sigma}$ map to eigenvectors of $D_{\sigma \circ \alpha}$, so the rotation operator $\alpha_{*}$ preserves this vector space. Up to completion there is thus a decomposition, natural in $\mathbb{T}$, of $T_{\sigma} L V$ as a sum

$$
\left.T_{\sigma} L V\right|_{[0,1)} \otimes\left(\oplus_{k \in \mathbb{Z}} \mathbb{C}(k)\right)
$$

where $\mathbb{C}(k)$ is the complex one-dimensional representation of the circle in which rotation by $\alpha$ acts as multiplication by $\exp (i k \alpha)$.

When the metric $h$ is flat this Fourier decomposition is a familiar construction (as it is over the space of constant loops), but the interval $[0,1)$ is not closed, so it is not clear that this decomposition behaves well as the loop varies. That question is the topic of this note.

1.3 The construction sketched above is classical, but it is usually formulated in terms of holonomy [1]: a loop $\sigma$ in $V$ defines a periodic map

$$
\tilde{\sigma}: \mathbb{R} \rightarrow \mathbb{R} / 2 \pi \mathbb{Z} \rightarrow V
$$

of the line to $V$, and if $v$ is an eigenvalue of $-i D_{\sigma}$ with eigenvalue $h$, then

$$
\tilde{v}(t)=v(t) \mathbf{e}(-h t / 2 \pi)
$$

(where $\mathbf{e}(t)$ abbreviates $\exp (2 \pi i t)$ ) is a section of the vector bundle $\tilde{\sigma}^{*} T V$ over the line, which satisfies the first-order differential equation $D_{\tilde{\sigma}} \tilde{v}=0$. This section is consequently determined by its initial conditions: clearly $\tilde{v}(0)=v(0)$, while

$$
\tilde{v}(2 \pi)=\mathbf{e}(-h) v(0) ;
$$


in other words, parallel transport (using the connection $\nabla$ ) of the vector $v(0)$ around the loop $\sigma$ results in a twist by $\mathbf{e}(-h)$. The space $\left.T_{\sigma} L V\right|_{[0,1)}$ spanned by eigenvectors of $-i D_{\sigma}$ with eigenvalues in $[0,1)$ is thus isomorphic to the tangent space $T_{\sigma(0)} V$ of $V$ at the basepoint $\sigma(0)$, by the map which assigns to an initial vector, its continuation by parallel transport around the loop. In this picture, $\exp \left(i D_{\sigma}\right)$ is the holonomy operator, and the correspondence $v \mapsto \tilde{v}$ sends eigenvectors of $D_{\sigma}$ to eigenvectors of holonomy. This justifies the claim made above, that $\left.T_{\sigma} L V\right|_{[0,1)}$ is finite-dimensional: in fact it has complex dimension $n$.

1.4 A choice of connection on $V$ thus assigns to a loop $\sigma$, a unitary automorphism of $T_{\sigma(0)} V$. This defines the classical holonomy map

$$
L V \rightarrow U_{n}
$$

which can also be interpreted as the composition

$$
L V \rightarrow L\left(B U_{n}\right) \cong U_{n} \times B U_{n} \rightarrow U_{n}
$$

obtained from the free loops on the classifying map for the complex vector bundle $T V$ (think of $B U_{n}$ as a universal space for complex vector bundles with Hermitian connection). Stably, the composition

$$
L V \rightarrow U_{n} \subset U \rightarrow U / O
$$

classifies the canonical polarization of $T L V$, that being an equivalence class [2 $\$ 2]$ of decompositions

$$
T L V=T_{+} L V \oplus T_{-} L V
$$

of the tangent bundle, generalizing the classical decomposition of Fourier modes into positive and negative frequencies.

Rotating the loop moves the basepoint, which changes the holonomy operator by conjugation. It follows that the quotient map to the space $\hat{U}_{n}$ of unitary conjugacy classes is invariant under $\mathbb{T}$-translation. The space of conjugacy classes in a connected Lie group is just the quotient of a maximal torus by the Weyl group, which in this case is the space $\mathbb{T}^{n} / \Sigma_{n}$ of unordered $n$-tuples of points on the circle.

1.5 The theorem of Hurewicz implies that

$$
\pi_{1}(L V) \cong H_{2}(V, \mathbb{Z}),
$$

so $L V$ will not be simply-connected in general. The $\mathbb{T}$-action on the loopspace lifts to an action on its universal cover, which commutes with the action of $H_{2}(V, \mathbb{Z})$ by covering transformations: consider the space of maps of a two-disk to $V$, modulo the equivalence relation which identifies two maps if they agree on their boundary circles, and if furthermore the map they then define, from a two-sphere to $V$, is nullhomotopic. The circle acts on this model by rotating the disks, while the second homotopy group of $V$ acts by attaching a bubble at the center of a disk.

The fixed-point set $L V^{\mathbb{T}}$ consists of constant loops, but the fixed point set of the circle action on the universal cover is a disjoint union of copies of $V$, indexed by 
$H_{2}(X, \mathbb{Z})$. The space of maps to a fibration is again a fibration, so the Hopf construction implies that $L S^{3}$ is a circle bundle over $\widetilde{L S}^{2}$; but the obvious circle action on the domanin has $S^{3}$ as its fixed-point set, so the circle action on the universal cover can be more complicated than one might think.

On fundamental groups the holonomy map induces the homomorphism $[2 \S 2]$

$$
b \mapsto 2 c_{1}(V) \cdot b: H_{2}(V, \mathbb{Z}) \rightarrow \mathbb{Z}
$$

defined by the Kronecker product with the first Chern class of $T V$; if the Chern class is rationally nontrivial, this defines an infinite cyclic cover $\widetilde{L V}$ and a map

$$
\widetilde{L V} \rightarrow \mathbb{R} \times S U / S O \rightarrow \mathbb{R}
$$

which is equivariant with respect to covering transformations, such that a generator $q$ of that group acts on $\mathbb{R}$ as translation by $d=\#\left(\operatorname{coker} 2 c_{1}(V)\right)$. I will call $\widetilde{L V}$ the holonomy cover of $L V$; I'm indebted to Graeme Segal, for suggesting that it is this cover, rather than the universal one, which is particularly interesting. It will be convenient to extend this terminology to include $L V$ itself as the holonomy cover, when the Chern class is (rationally) trivial.

\section{Morton's logarithm}

2.0 The main result of this paragraph is the construction of a section

$$
\widetilde{\log }: \widetilde{\mathrm{SP}}_{n}(\mathbb{T}) \rightarrow \mathrm{SP}_{n}(\mathbb{R})
$$

of the quotient map from the space of unordered points on the line, to the universal cover of the corresponding space of points on the circle. Soon after it was written, I learned from Elmer Rees that it duplicates the argument of [3], published in 1967. As the construction is not very long, I have left it unchanged.

2.1 The $n$th symmetric power $\operatorname{SP}_{n}(X)$ of a space $X$ is the quotient $X^{n} / \Sigma_{n}$ of the $n$-fold cartesian product of copies of $X$ by the symmetric group $\Sigma_{n}$. The action of the symmetric group on $\mathbb{R}^{n}$ defined by permuting coordinates has an invariant codimension one subspace $\mathbb{R}_{0}^{n-1}$ consisting of vectors whose coordinates sum to zero, so we can decompose the symmetric power of the real line as a product

$$
\mathrm{SP}_{n}(\mathbb{R}) \cong \mathbb{R}_{0}^{n-1} / \Sigma_{n} \times \mathbb{R},
$$

where projection onto the last coordinate sends an $n$-tuple of real numbers to its average. Let

$$
\mathbf{v} \mapsto \sum\left\{v_{k}\right\}: \mathbb{R}^{n} \rightarrow \mathrm{SP}_{n}(\mathbb{R})
$$

denote the quotient map. The exponential

$$
t \mapsto \mathbf{e}(t): \mathbb{R} \rightarrow \mathbb{R} / \mathbb{Z}=\mathbb{T}
$$

from the line to the circle defines a map

$$
\text { e }: \mathrm{SP}_{n}(\mathbb{R}) \rightarrow \mathrm{SP}_{n}(\mathbb{T})
$$


which sends the configuration $\sum\left\{v_{k}\right\}$ in $\mathbb{R}$ to the configuration $\sum\left[v_{k}\right]$ in $\mathbb{T}$. The summation map

$$
\sum\left[v_{k}\right] \mapsto\left[\sum v_{k}\right]: \mathrm{SP}_{n}(\mathbb{T}) \rightarrow \mathbb{T}
$$

is a homotopy equivalence, for $\mathbb{T}$ is homotopy-equivalent to $\mathbb{C}^{\times}$, and the map

$$
\left\{z_{i}\right\} \in \mathbb{C}^{\times} \mapsto \prod_{[1 \leqslant i \leqslant n}\left(z-z_{i}\right): \mathrm{SP}_{n}\left(\mathbb{C}^{\times}\right) \rightarrow \mathbb{C}^{n-1} \times \mathbb{C}^{\times}
$$

which assigns to an unordered $n$-tuple of nonvanishing complex numbers, the monic polynomial with those elements as its zeros, is a homeomorphism. It follows that the universal cover

$$
\widetilde{\mathrm{SP}}_{n}(\mathbb{T})=\left\{\left(\sum\left[t_{k}\right], t\right) \in \mathrm{SP}_{n}(\mathbb{T}) \times \mathbb{R} \mid t \equiv \sum t_{k} \bmod \mathbb{Z}\right\}
$$

of $\mathrm{SP}_{n}(\mathbb{T})$ splits, via the map

$$
\left(\sum\left[t_{k}\right], t\right) \mapsto\left(\sum\left[t_{k}-t / n\right], t\right): \widetilde{\mathrm{SP}}_{n}(\mathbb{T}) \cong \mathrm{SP}_{n}^{0}(\mathbb{T}) \times \mathbb{R}
$$

as a product.

2.2 A point of the standard simplex $\Delta^{n-1}$ is an ordered $n$-tuple $\mathbf{x}=\left(x_{1}, \ldots, x_{n}\right)$ of real numbers between zero and one, subject to the constraint

$$
\sum x_{k}=1
$$

if we define

$$
m(x)=n^{-1} \sum_{1 \leqslant k \leqslant n} k x_{k},
$$

then the sequence $t_{1}=m(x), t_{2}=x_{1}+m(x), \ldots, t_{n-1}=x_{n-2}+\ldots+x_{1}+$ $m(x), t_{n}=m(x)-x_{n}$ of real numbers satisfies

$$
\sum t_{i}=n-1
$$

with nonnegative interpoint distances

$$
t_{2}-t_{1}=x_{1}, t_{3}-t_{2}=x_{2}, \ldots, t_{1}-t_{n}=x_{n} .
$$

If $\sigma(\mathbf{x})=\left(x_{2}, \ldots, x_{n}, x_{1}\right)$ then

$$
m(\sigma(x))=m(x)+x_{1}-1 / n,
$$

so the sequence

$$
m(x)+x_{1}-1 / n, m(x)+x_{2}+x_{1}-1 / n, \ldots, m(x)-1 / n
$$

associated to $\sigma(\mathbf{x})$ differs by a cyclic shift and a translation by $1 / n$ from the sequence defined by $\mathbf{x}$.

2.3 Let

$$
\mathbf{x} \mapsto \lambda(\mathbf{x})=\sum\left\{t_{k}\right\}: \Delta^{n-1} \rightarrow \mathrm{SP}_{n}(\mathbb{R})
$$


denote the result of forgetting the order on the points in this construction. Its composition

$$
\mathbf{x} \mapsto \mathbf{e}(\lambda(\mathbf{x})): \Delta^{n-1} \rightarrow \mathrm{SP}_{n}^{0}(\mathbb{T})
$$

with the exponential takes values in the subspace of configurations on the circle which sum to zero. The interior of the simplex maps onto the subspace of zero-sum configurations with multiplicities at most one: indeed, any configuration of $n$ distinct points on the circle defines a sequence $\tau_{1}, \ldots, \tau_{n} \in \mathbb{T}$, ordered counterclockwise from zero, with interpoint distances $x_{k}=\tau_{k+1}-\tau_{k}$ defining a point $\mathbf{x}$ in the interior of the simplex. If we interpret these $\tau$ 's to be real numbers in the unit interval, then

$$
m(x)=n^{-1} \sum_{1 \leqslant k \leqslant n-1} k\left(\tau_{k+1}-\tau_{k}\right)+\left(\tau_{1}-\tau_{n}\right)
$$

can be rewritten as

$$
\tau_{1}+n^{-1}\left[\sum_{1 \leqslant k \leqslant n}(k-1) \tau_{k}-\sum_{1 \leqslant k \leqslant n} k \tau_{k}\right]=\tau_{1}-n^{-1} \sum_{1 \leqslant k \leqslant n} \tau_{k} ;
$$

thus $\mathbf{e} \circ \lambda$ applied to $\mathbf{x}$ recovers the original configuration, up to a translation by $n^{-1} \sum \tau_{k}$. The sum of the $\tau$ 's is by assumption an integer $m$, so the cyclic shift $\sigma^{m}(\mathbf{x})$ recovers the original configuration.

A similar argument shows that $\mathbf{e} \circ \lambda$ is one-to-one on the interior of the simplex. Its faces are defined by the vanishing of various barycentric coordinates; these faces map to configurations with points of multiplicity greater than one, so degenerate configurations which partition $n$ into $p$ parts correspond to faces of codimension $n-p$. By induction the map is a bijection on these open faces, and is hence a bijection on the whole of $\Delta^{n-1}$.

2.4 It follows that the (well-defined!) composition

$$
\lambda \circ[\mathbf{e} \circ \lambda]^{-1}: \mathrm{SP}_{n}^{0}(\mathbb{T}) \rightarrow \mathrm{SP}_{n}(\mathbb{R})
$$

is a section of the map e restricted to the zero-sum configurations on the circle; it defines an analog of the logarithm. If

$$
\widetilde{\log _{0}}: \mathrm{SP}_{n}^{0}(\mathbb{T}) \rightarrow \mathbb{R}_{0}^{n-1} / \Sigma_{n}
$$

denotes its composition with projection onto the first factor of $\mathrm{SP}_{n}(\mathbb{R})$, then the equation

$$
\widetilde{\log }_{0}\left(\sum\left[t_{k}\right]\right)=\sum\left\{t_{k}\right\}
$$

makes a certain amount of sense, and $\widetilde{\log }_{0}$ extends to define a section

$$
\widetilde{\log }\left(\sum\left[t_{k}\right], t\right)=\left(\sum\left\{t_{k}-t / n\right\}, t / n\right): \widetilde{\mathrm{SP}}_{n}(\mathbb{T}) \rightarrow \mathrm{SP}_{n}(\mathbb{R})
$$

of the lift

$$
\sum\left\{v_{k}\right\} \mapsto \tilde{\mathbf{e}}\left(\sum\left\{v_{k}\right\}\right)=\left(\sum\left[v_{k}\right], n v\right): \mathrm{SP}_{n}(\mathbb{R}) \rightarrow \widetilde{\mathrm{SP}}_{n}(\mathbb{T})
$$

of $\mathbf{e}$ to the universal cover ( $v$ being the average of the $v_{k}$ ). 
The map $\mathbf{e}$ is equivariant with respect to the translation actions of $\mathbb{R}$ and $\mathbb{T}$ on $\mathrm{SP}_{n}(\mathbb{R})$ and $\mathrm{SP}_{n}(\mathbb{T})$, so its lift $\tilde{\mathbf{e}}$ is $\mathbb{R}$-equivariant as well. The composition e $\circ \lambda$ (or, more correctly, its inverse) is essentially just an identification of the quotient of $\mathrm{SP}_{n}(\mathbb{T})$ by the circle action, so it is unreasonable to hope that the sections constructed here might be $\mathbb{R}$-equivariant.

Nevertheless, the map $\widetilde{\log }$ is equivariant with respect to translation by the subgroup $\mathbb{Z}$ of $\mathbb{R}$.

\section{A decomposition of the tangent bundle}

3.1 Suppose, then, that $V$ is a connected and simply-connected almost complex manifold of real dimension $2 n$. The holonomy of the connection associated to a Hermitian metric on $V$ defines a $\mathbb{T}$-invariant map

$$
\hat{H}: L V \rightarrow \hat{U}_{n}=\mathrm{SP}_{n}(\mathbb{T}),
$$

and thus a lifting

$$
\tilde{H}: \widetilde{L V} \rightarrow \widetilde{\mathrm{SP}}_{n}(\mathbb{T})
$$

to a map of covers. The composition

$$
\eta=\widetilde{\log } \circ \tilde{H}: \widetilde{L V} \rightarrow \mathrm{SP}_{n}(\mathbb{R})
$$

continuously assigns to an element $\sigma^{\prime}$ of the cover, a choice of $n$ real eigenvalues of $-i D_{\sigma}$, whose eigenvectors (up to suitable twists by $\mathbb{C}(k)$ ) span $\left.T_{\sigma} L V\right|_{[0,1)}$. Let

$$
\sigma^{\prime} \mapsto \Pi_{\sigma^{\prime}}:=\oplus_{h \in \eta\left(\sigma^{\prime}\right)} \mathcal{P}_{h}: T_{\sigma^{\prime}} \widetilde{L V} \rightarrow T_{\sigma^{\prime}} \widetilde{L V}
$$

be the function which assigns to $\sigma^{\prime}$, the projection onto the span of the eigenvectors supported in $\eta\left(\sigma^{\prime}\right)$. This is a continuous $\mathbb{T}$-equivariant family of projections, and its image

$$
\mathbf{T} V=\text { Image } \Pi \subset T \widetilde{L V}
$$

is a T-equivariant complex $n$-plane bundle over $\widetilde{L V}$. The equivariant bundle monomorphism

$$
\mathbf{T} V \otimes\left(\oplus_{k \in \mathbb{Z}} \mathbb{C}(k)\right) \rightarrow T \widetilde{L V V}
$$

is a global analog of the local Fourier expansion in $\S 1.2$.

3.2 Covering translations will not preserve this decomposition, but $\S 2.4$ implies the existence of an equivariant isomorphism

$$
q^{*} \mathbf{T} V \cong \mathbf{T} V \otimes \mathbb{C}(d),
$$

such that the extension $[6 \S 6.5]$

$$
1 \rightarrow \mathbb{C}^{\times} \rightarrow H \rightarrow \mathbb{Z} \times \mathbb{T} \rightarrow 0
$$

defined by the bilinear form

$$
\left(k_{0}, c_{0}\right),\left(k_{1}, c_{1}\right) \mapsto c_{0}^{k_{1}} c_{1}^{-k_{0}}
$$


on $\mathbb{Z} \times \mathbb{T}$ acts on the polarization

$$
T_{ \pm} \widetilde{L V}=\mathbf{T} V \otimes\left(\oplus_{k \leqslant, \geqslant 0} \mathbb{C}(k)\right),
$$

by a lift of the action of $\mathbb{Z} \times \mathbb{T}$ on $\widetilde{L V}$.

Acknowledgements This note began in conversations with Ralph Cohen during the August 2000 conference on equivariant homotopy theory at Stanford. I also owe a great deal to conversations with Matthew Ando, and to Tom Goodwillie and Graeme Segal (for trying to straighten out my thinking about holonomy). I owe Jim Martino ten million dollars in thanks for help with MAPLE calculations at a crucial stage in this project. I would also like to thank Clarence Wilkerson and Bill Dwyer for encouraging correspondence, and Elmer Rees for telling me about [3,4]. That this note exists at all is due to Tom Mrowka, who saw that I had mistakenly assumed [e.g. in the case $V=S^{2}$ ] that the holonomy map was inessential; many thanks to him for taking the matter seriously.

\section{References}

[1] M. Berger, Sur les groupes d'holonomie homogéne des variétés a connexion affine et des variétés riemanniennes, Bull. Soc. Math. France 83 (1955) 279 330

[2] R.L. Cohen, J.D.S. Jones, G.B. Segal, Floer's infinite dimensional Morse theory and homotopy theory, in The Floer Memorial Volume, Birkhäuser, Progress in Mathematics 133 (1995) 297-326

[3] H.R. Morton, Symmetric products of the circle, Proc. Cambridge Phil. Soc. 63 (1967) 349-352

[4] J. Mostovoy, Geometry of truncated symmetric products and real roots of real polynomials, Bull. London Math. Soc. 30 (1998) 159-165

[5] R. Palais, Foundations of global nonlinear analysis, Benjamin (1968)

[6] C. Voisin, Mirror Symmetry, SMF/AMS Texts (1999)

[7] R.O. Wells, Differential analysis on complex manifolds, Springer GTM $65(1980)$

This article may be accessed via WWW at http://www.rmi.acnet.ge/hha/ or by anonymous ftp at

ftp://ftp.rmi.acnet.ge/pub/hha/volumes/2001/n2a7/v3n2a7.(dvi,ps,pdf)

Jack Morava jack@math.jhu.edu

Department of Mathematics

Johns Hopkins University

Baltimore, Maryland 21218 